\title{
Variation of Platelet Indices in Differential Diagnosis of Thrombocytopenia - An Observational Study in a Tertiary Care Centre
} Debaditya Samanta $^{1 *}$, Asim Kumar Manna ${ }^{2}$

${ }^{1}$ Post Graduate Trainee, ${ }^{2}$ Professor, Department of Pathology, Institute of Post Graduate Medical Education \& Research, Kolkata

DOI: $10.36347 /$ sjams.2020.v08i11.040

| Received: 12.11.2020 | Accepted: 23.11.2020 | Published: 28.11.2020

*Corresponding author: Debaditya Samanta

Abstract

Differential diagnosis of thrombocytopenia can be assessed by studying variation of platelet indices. Different platelet indices such as MPV, PDW, P-LCR, Plateletcrit can be used for these purposes. A study of 200 cases of thrombocytopenia were conducted, corroborated there causes with different platelet indices. It was observed that in the hyper-destructive group (viz. due to infective causes, ITP and SLE), MPV, PDW and P-LCR showed an inverse correlation with the platelet count. Whereas, in the hypo-productive group (viz. due to aplastic anaemia, megaloblastic anaemia, and acute leukaemia and drug-induced) and in control group all the parameters showed a direct correlation to their respective platelet counts. However, bone marrow is the gold standard to differentiate between hypo-productive and hyper-destructive thrombocytopenia, it is agreed that more elaborate and controlled studies are needed to ascertain the importance of these readily available platelet indices in term of their sensitivity, specificity, positive and negative predictive values to get a proper idea about the underlying patho-mechanism of thrombocytopenia. These parameters are to be viewed as a guide to understand the basic patho-mechanisms of thrombocytopenia.

Keywords: Thrombocytopenia, platelet indices, MPV, PDW, PLCR, Plateletcrit.

Copyright $\odot$ 2020 The Author(s): This is an open-access article distributed under the terms of the Creative Commons Attribution 4.0 International License (CC BY-NC 4.0) which permits unrestricted use, distribution, and reproduction in any medium for non-commercial use provided the original author and source are credited.

\section{INTRODUCTION}

Platelets were first described in 1841 by Addison as "extremely minute.... granules" [1] in clotting blood and were termed as platelets (blutplattchen) by Bizzozero, who also observed their adhesive qualities as "increased stickiness when a vascular wall is damaged." [2]. The same elements were identified by microscopic examination of blood smears by Osler and Schaefer and Hayem in the late nineteenth century [3, 4]. Duke, a student of James Homer Wright's demonstrated association of low platelet count, or thrombocytopenia, with clinical bleeding that could be corrected by transfusion[5].

Platelets are small fragments of cytoplasm derived from megakaryocytes. They are small anucleate cells (approx. 1.5-3.5 $\mu \mathrm{m}$ in diameter) which play an important role in haemostasis, serving to plug holes in blood vessels and initiating coagulation cascade. To serve these purposes, platelets have certain unique properties, including the ability to adhere to injured blood vessels and to other platelets, undergo dramatic shape changes, release granules containing vasoactive and thrombogenic substances, and modulate the phospholipid content of their external membrane. Platelets also release substances that promote wound healing. Normal platelet count is between $280 \times 10^{9} / \mu 1$ of blood [6]. Megakaryocytes are large polypoid cells in the bone marrow that produce circulating platelets. Each megakaryocyte produces about 1000-4000 platelets. They represent about $0.05 \%$ of nucleated cells in bone marrow. Howell coined the term megakaryocyte as early as 1890 but it was not until 1906 that James Homer Wright put forth the hypothesis that blood platelets are derived from the cytoplasm of megakaryocytes and the basis of thrombopoiesis was established [7, 8].

Mean Platelet Volume (MPV) is a machine calculated measurement describing the average size of platelets in blood. It provides an indicator of whether the bone marrow is manufacturing platelets normally. Normal MPV ranges approximately 6.8-10.4 fl [9-11] MPV increases when many large platelets are present. Young platelets are usually larger in size. Platelets tend to swell during the first 2 hours in EDTA anticoagulant, shrinking again with longer storage. MPV increases in ITP, Bernard-Soulier disease, May-Hegglin anomaly, sepsis (recovery phase), CML, MDS, DIC, TTP infection related thrombocytopenia like dengue and malaria etc. MPV decreases in aplastic anaemia, Wiskott-Aldrich syndrome, TAR syndrome, storage pool disease, hypersplenism [12]. 
Platelet Distribution Width (PDW) is analogous to the Red Cell Distribution Width (RDW) and compares the uniformity and heterogeneity of platelet size and is a measure of platelet anisocytosis and standard PDW ranges from 9-14 fl [9-11] Increased PDW values are observed in essential thrombocytopenia, aplastic anaemia, megaloblastic anaemia, and chemotherapy. The increased PDW values in such cases may be due to dysfunctional megakaryocyte development. If erythrocyte fragments are being counted as platelets, the PDW will be falsely elevated as it broadens the platelet volume distribution curve. It is a good tool to differentiation between essential thrombocytopenia from reactive thrombocytosis [12].

Platelet-Large Cell Ratio (P-LCR) is a machine calculated parameter which is defined as number of platelets falling above the threshold on the platelet size histogram divided by the total number pf platelets. A high P-LCR may indicate peripheral immune destruction of platelets [9-11].

Plateletcrit (PCT) is the volume percentage that platelets match on a total volume of blood and is directly related to the number of platelets and MPV. Normal platelet count has a plateletcrit between 0.20 and $0.36 \%$ [9-11].

Thrombocytopenia defined as platelet count less than 1,50000/ $\mu 1$ of blood. Grade 1: platelet count of $75000-1,50000 / \mu 1$. Grade 2: platelet count of 50000$75000 / \mu 1$. Grade 3: platelet count of $2500-50000 / \mu 1$. Grade 4: platelet count of $<25000 / \mu 1$ [13]. Decreased production of platelets (hypo-productive) results due to selective impairment of platelet production as in drug induced, or due to nutritional deficiencies or due to bone marrow failure syndrome and also due to bone marrow replacement as in leukaemia, MDS. Decreased platelet (hyper-destructive) survival results due to immunologic destruction or drug induced or any other nonimmunologic destruction as in DIC [14]

In general, platelets are large when thrombocytopenia results from increased destruction and small with disorder of diminished production. We aimed to study and to find out whether these simple and readily available platelet indices can guide us to differentiate between hypo-productive and hyperdestructive causes of thrombocytopenia and can help in clinical and therapeutic decision making by narrowing down the differential diagnosis. Though there is some amount of research on the topic throughout the world, yet there is little data available regarding same in eastern India. control. Study variables were selected as age, sex, clinical history especially fever, cytopenia, bleeding manifestations like purpuric spots, history of alcohol consumption, drug history especially cytotoxic drugs like methotrexate, history of any chemo or radiotherapy, history of any blood transfusion. MPV, Plateletcrit (PCT), Platelet distribution width (PDW), PlateletLarge Cell Ratio (P-LCR) and bone marrow examination variables were analysed among the study samples. After taking clinical history blood samples were drawn and platelet count estimated by Sysmex automated cell counters XE-2100/ XT-4000i. The obtained platelet counts were corroborated with manual smear count which was stained by Leishman's stain. Collected data was summarized by routine descriptive statistics viz. mean and standard deviation for normally distributed numerical variables and counts and percentages for categorical variables. Numerical variables were compared between groups by Student Independent Sample T-test. Association between numerical values was calculated by Pearson's correlation coefficient. Key variables were expressed with a 95\% confidence interval (CI) and $p$ value $<0.05$ was considered statistically significant.

\section{RESULTS AND ANALYSIS}

We intended to compare the various readily available platelet indices viz. MPV, PCT, PDW and PLCR obtained from automated cell counter and saw if we could use them in differentiating between hypoproductive and hyper-destructive causes of thrombocytopenia.

Among 52 hypo-productive causes of thrombocytopenia $(26 \%)$, in our current setting we observed 7 cases of aplastic/hypoplastic anaemia (3.5\%), 10 cases of megaloblastic anaemia (5\%), 22 cases of acute leukaemia (11\%) and 13 cases of drug induced thrombocytopenia (6.5\%). Among the 148 hyper-destructive causes of thrombocytopenia (74\%), in our current setting we observed 117 cases of infective or secondary immune-mediated thrombocytopenia $(58.5 \%), 26$ cases of primary ITP $(13 \%)$ and 5 cases of thrombocytopenia due to SLE (2.5\%) [Box 1, Fig 1].

Table-1: Different causes of thrombocytopenia.

\begin{tabular}{|l|l|l|l|}
\hline Hypo-Productive & $\begin{array}{l}\text { No of } \\
\text { Cases }\end{array}$ & $\begin{array}{l}\text { Hyper- } \\
\text { Destructive }\end{array}$ & $\begin{array}{l}\text { No of } \\
\text { Cases }\end{array}$ \\
\hline Aplastic Anaemia & 07 & Infective & 117 \\
\hline $\begin{array}{l}\text { Megaloblastic } \\
\text { Anaemia }\end{array}$ & 10 & ITP & 26 \\
\hline Acute Leukaemia & 22 & SLE & 05 \\
\hline Drug-induced & 13 & & \\
\hline Total & 52 & & 148 \\
\hline
\end{tabular}

\section{Methods ANd Materials}

We conducted a cross-sectional observational study with a sample size of 200 samples having platelet count $<1,50000 / \mu 1$. We also took 200 individuals as 


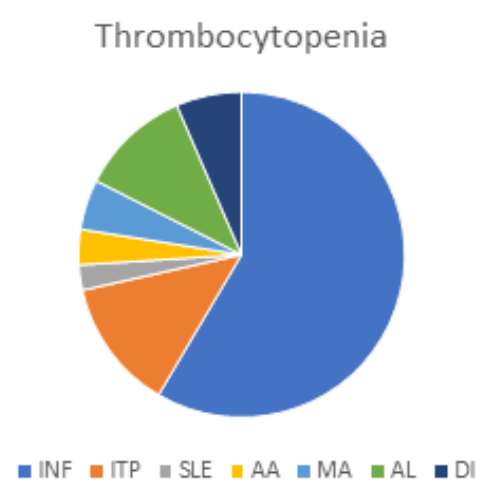

Fig-1: Different causes of thrombocytopenia represented in a piediagram

In the present study, the ages of both the patient and the control groups have been divided into nine groups ranging from 0 to 90 years. Maximum number of patients seemed to fall in the 21 to 30 years age bracket, followed by 11 to 20 years bracket. On the other hand maximum number of controls belonged to 31 to 40 years age category followed by 11 to 20 years age bracket [Fig 2].

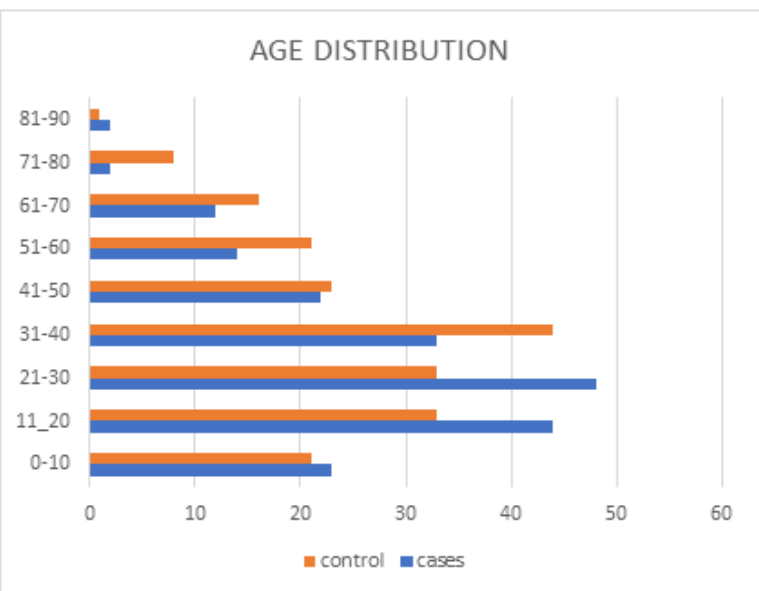

Fig-2: Age distribution represented in bar-diagram

In the present study there were 122 males and 78 females in the patient group, whereas 120 males and 80 females in the control group [Fig 3].

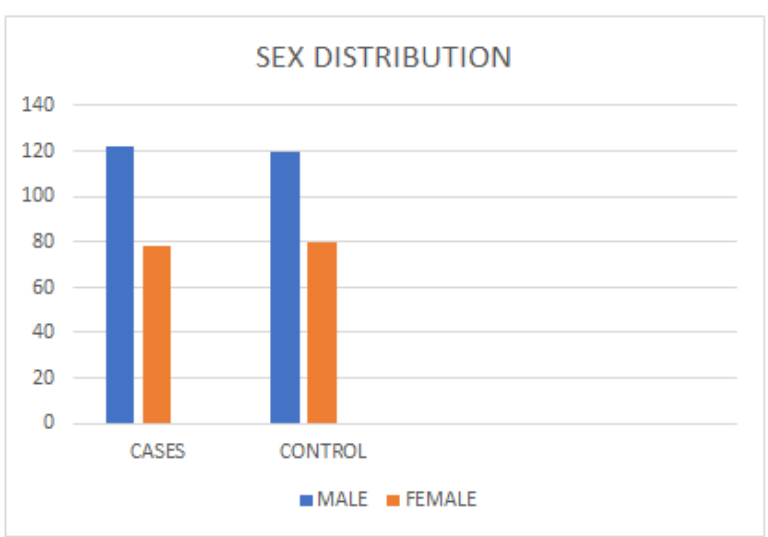

Fig-3: Sex distribution of cases and controls
In the control group, the highest and lowest values of MPV were 13.2 and $5.6 \mathrm{fl}$ respectively $(9.3 \pm 1.23)$, while in the hyper-destructive group which includes the infective causes, primary immunological causes and SLE, they were 15.9 and $6.1 \mathrm{fl}$ respectively and, in the the hypo-productive group which includes aplastic anaemia, megaloblastic anaemia, acute leukaemia and drug-induced thrombocytopenia, they were 7.8 and $5.1 \mathrm{fl}$ respectively. In this study it was seen that barring the thrombocytopenic patients diagnosed with SLE, in all other groups the MPV was found to be statistically significant when compared with control group. The MPV of both the broader etiological groups viz. hypo-productive (AA, MA, AL, DR) and hyper-destructive (INF, ITP, SLE) are mostly statistically significant $(\mathrm{p}<0.05)$ when pitted against each other. The highest values and lowest value of plateletcrit (PCT) in the control group are $0.42 \%$ and $0.16 \%$ respectively $(0.26 \pm 0.056)$, while in the hyperdestructive group they are $0.21 \%$ and $0.09 \%$ respectively and in hypo-productive group they are $0.18 \%$ and $0.1 \%$ respectively. Only the control group, which mostly had normal range of plateletcrit, has statistic when compared to both the hypo-productive and hyper-destructive groups. The hypo-productive and the hyper-destructive groups, on the other hand, when compared did not give a consistent statistically significant value and were rather dependent on specific aetiology of the cause. The highest values and lowest value of PDW in the control group are 17.1 and $9.3 \mathrm{fl}$ respectively $(12.5 \pm 1.59)$, while in the hyper-destructive group they are 18 and $12.7 \mathrm{fl}$ respectively and in hypoproductive group they are 15.6 and $10.4 \mathrm{fl}$ respectively. It was noted only the PDW of the hyper-destructive group was statistically significant when compared to that of the control group. The hypo-productive group, with the exception of drug induced causes of thrombocytopenia, was not statistically significant when compared to the control group. The highest values and lowest value of P-LCR in the control group are 37.5 and $16.3 \mathrm{fl}$ respectively $(25.4 \pm 3.85)$, while in the hyperdestructive group they are 36.3 and $23.8 \mathrm{fl}$ respectively and in hypo-productive group they are 30.6 and $16.9 \mathrm{fl}$ respectively.

In the study it was that only the P-LCR of the hyper-destructive group was statistically significant when compared to that of control group. The hypoproductive group, with the exception of drug induced causes of thrombocytopenia, was not statistically significant when compared group. It was observed that in the hyper-destructive group, MPV, PWD and P-LCR had moderately negative $r$ values with respect to platelet counts, that is the MPV, PDW and P-LCR in the hyper-destructive group showed an inverse correlation with the platelet count. Whereas, in the hypo-productive group and in the control group all the parameters showed positive $r$ values, which mean a direct correlation to their respective platelet counts. 
Table-2

\begin{tabular}{|l|l|l|l|l|l|l|l|l|}
\hline & CON & INF & ITP & SLE & AA & MA & AL & DI \\
\hline MPV & $9.3 \pm 1.23$ & $13.04 \pm 1.13$ & $13.28 \pm 1.96$ & $10.12 \pm 3.2$ & $5.51 \pm 1.24$ & $5.94 \pm 0.29$ & $6.02 \pm 0.3$ & $6.37 \pm 0.64$ \\
\hline PCT & $0.26 \pm 0.056$ & $0.15 \pm 0.025$ & $0.13 \pm 0.021$ & $0.16 \pm 0.005$ & $0.1 \pm 0.005$ & $0.16 \pm 0.024$ & $0.13 \pm 0.021$ & $0.15 \pm 0.022$ \\
\hline PDW & $12.5 \pm 1.59$ & $15.67 \pm 0.75$ & $16.08 \pm 0.75$ & $15.18 \pm 1.72$ & $11.71 \pm 0.94$ & $13.03 \pm 0.72$ & $12.86 \pm 0.99$ & $13.66 \pm 0.98$ \\
\hline P-LCR & $25.4 \pm 3.85$ & $30.47 \pm 1.83$ & $32.06 \pm 1.3$ & $30.66 \pm 1.79$ & $25.47 \pm 2.68$ & $27.11 \pm 2.17$ & $25.59 \pm 3.61$ & $28.03 \pm 1.36$ \\
\hline
\end{tabular}

Box 2: Comparison of the average values $( \pm 2$ $\mathrm{SD})$ of the platelet indices according to aetiology in patients and in control groups. [CON-Control, INFInfective, ITP-Immune thrombocytopenic purpura,
SLE-Systemic Lupus Erythematous, AA-Aplastic anaemia, MA-Megaloblastic anaemia, AL- Acute leukaemia, DI-Drug induced].

Table-3

\begin{tabular}{|l|l|l|l|l|}
\hline & MPV & PCT & PDW & P-LCR \\
\hline Hyper-destructive & -0.497 & 0.788 & -0.385 & -0.411 \\
\hline Hypo-productive & 0.415 & 0.828 & 0.372 & 0.194 \\
\hline CONTROL & 0.395 & 0.752 & 0.236 & 0.375 \\
\hline
\end{tabular}

Box 3: Pearson's correlation coefficient $(r)$ values for all the platelet indices in hyper-destructive and hypo-productive causes of thrombocytopenia and in non-thrombocytopenic control group when pitted against their respective platelet counts. (-1: perfect negative linear, -0.7 : strong negative linear, -0.5: moderate negative, -0.3 : weak negative linear, 0 : no linear, +0.3: weak positive linear, +0.5 : moderate positive, +0.7: strong positive linear, +1 : perfect positive linear correlation) [15].

\section{DISCUSSION}

It is important to differentiate between thrombocytopenia caused by hypo-production of platelets from that caused by hyper-destruction or to know which is more dominant. Measurement of the platelet indices by using automated-haematologicalanalyzer is simple, inexpensive, quick test and obivate examiner bias, also avoid changes in platelets diameter that occur due to enhanced adhesiveness with flattening and increasing size of the platelets on the glass slide during blood smears and the changes that result from delayed time between venipuncture and preparation of smears. However, it is not always possible to measure platelet indices such as in severe thrombocytopenia and in red cell fragmentation because a platelet histogram cannot be appropriately drawn and the platelet indices cannot be measured.

In this study such obstacles did not occur and the platelet indices revealed that the MPV, PDW, P-LCR were significantly higher in hyper-destructive thrombocytopenia (due to infective causes, ITP, SLE) than hypo-productive thrombocytopenia (aplastic anaemia, megaloblastic anaemia, acute leukaemia and cytotoxic drug-induced thrombocytopenia) ( $p$ value <0.05). The high MPV in hyper-destructive thrombocytopenia could be explained by the fact that newly produced platelets are larger than circulating platelets, which tend to decrease in size with age in the circulation over the 7-10-day platelet lifespan. As a result, in patients with thrombocytopenia secondary to peripheral destruction the MPV is increased, reflecting active bone marrow compensation with release of young platelets "left shifted". Also, a significantly higher PDW in hyper-destructive thrombocytopenia was also reported in this study ( $p$ value $<0.05$ ). Again, the increased bone marrow production of platelets is associated with heterogenic population of platelet (platelet anisocytosis). PCT was not significantly different in both groups similarly no study considers it as valuable index to differentiate between hypoproductive and hyper-destructive thrombocytopenia, PCT represent a volume percent of platelets and its value is a result of PDW multiplied by platelet count so it is affected by the severity of thrombocytopenia of any cause [16]. However, PCT can be used as an effective screening tool to detect quantitative abnormalities of platelet (for both thrombocytopenia and thrombocytosis) as is evident by its strong positive correlation with platelet count.

\section{Conclusion}

In the present study it was observed that in the hyper-destructive group (viz. due to infective causes, ITP and SLE), MPV, PDW and P-LCR showed an inverse correlation with the platelet count. Whereas, in the hypo-productive group (viz. due to aplastic anaemia, megaloblastic anaemia, and acute leukaemia and druginduced) and in control group all the parameters showed a direct correlation to their respective platelet counts. However, as one 2013 study concluded that MPV provides a small insight to the aetiology of thrombocytopenia limited by insufficient sensitivity and specificity and bone marrow is the gold standard to differentiate between hypo-productive and hyperdestructive thrombocytopenia [17] it is agreed that more elaborate and controlled studies are needed to ascertain the importance of these readily available platelet indices in term of their sensitivity, specificity, positive and negative predictive values to get a proper idea about the underlying patho-mechanism of thrombocytopenia so that it can help the clinicians in better decision making. These parameters are to be viewed as a guide to understand the basic patho-mechanisms of 
thrombocytopenia and not to be used for definitive diagnosis in a case of disease. Bone marrow examination remains the gold standard for find the exact aetiology. So, we see that proper interpretation of the platelet parameters is of immense important to get an idea about the patho-mechanisms of thrombocytopenia. An elaborate study is suggested to get more authentic information in this field.

\section{REFERENCES}

1. Addison W. On the colourless corpuscles and on the molecules and cytoblasts in the blood. London Med Gaz. 1841; NS30:144-152.

2. Bizzozero G. Ueber einer neuren formblesandtheil des blutes und dessen rolle bei der thrombose und der blutegerinnung. Virchow's Arch Pathol Anat Physiol. 1882; 90:261-332.

3. Osler W, Schaefer EA, Uber einige im blute vorhandene bacterienbildende massen. Centre Med Wissensch. 1873;11:577-578.

4. Hayem G. Research's sur l'evolution des hematics dans le sang de l'homme, et des vertebres. Arch Physiol (deuxieme serie). 1878;692-734.

5. Duke W. The relation of blood platelets to hemorrhagic disease. Description of a method for determining the bleeding time and the coagulation time and report of 3 cases hemorrhagic disease relieved by transfusion. JAMA. 1910; 55:11851192.

6. Bates I, Lewis SM. Reference ranges and normal values. In Bain BJ, Bates I, Laffan MA, Lewis SM (ed). Dacie and Lewis Practical Haematology, $11^{\text {th }}$ Edition. Elsevier; 2012;14.

7. Wright J. The origin and nature of the blood platelets. Boston Med J 1906; 23:643-645.
8. Wright J. The histogenesis of blood platelets. J Morphol. 1910; 21:117-130.

9. Bates I, Lewis SM. Basic haematological techniques. In Bain BJ, Bates I, Laffan MA, Lewis SM (ed). Dacie and Lewis Practical Haematology, $11^{\text {th }}$ Edition. Elsevier. 2012; 47-48.

10. Baur JD. Clinical Laboratory Methods, $9^{\text {th }}$ Edition. Mosby. 1982; 328.

11. Turgeon $\mathrm{ml}$. Clinical haematology: theory and procedures, $3^{\text {rd }}$ Edition: 342 .

12. Lokwani DP. Platelets. The ABC of CBC: Interpretation of complete blood count and histograms, $1^{\text {st }}$ edition, Jaypee. New Delhi.2013; 59-60.

13. Bleeding disorders: Haemorrhagic Diatheses. In Kumar V, Abbas AK, Aster JC (ed). Robbins and Cotron Pathologic Basis of Diseases, $10^{\text {th }}$ Edition. Elsevier. 2020; 657-58.

14. Je Hoon Lee, MD. Clinical Significance of Platelet Indices in Haematological Disorders. Korean J Clin Pathol. 1997; 17(2):201-8.

15. How to interpret a correlation coefficient. http://www.dummies.com/education/math/statistics /how-to-interpret-a-correlation-coefficient-r/

16. Martin C, Riddle P, Jones A. What is mean platelet volume?. 2013. Available from: www.wisegeek.com/what-is-mean-plateletvolume.htm.

17. Ruchee Khanna, Deepak Nayak M, Chetan Mnohar, Murli Dhar. A retrospective evaluation of mean platelet volume as a discriminating factor in thrombocytopenia of hypoproductive and hyperdestructtive aeitologies. Journal of Evaluation of Medical and Dental Sciences. 2013;2(47):905965. 